\title{
DOMINIQUE KALIFA, Tu entreras dans le siècle en lisant
}

\section{Fantômas}

\section{Francesca Forcolin}

\section{(2) OpenEdition}

\section{Journals}

\section{Edizione digitale}

URL: http://journals.openedition.org/studifrancesi/15727

DOI: 10.4000/studifrancesi. 15727

ISSN: 2421-5856

\section{Editore}

Rosenberg \& Sellier

\section{Edizione cartacea}

Data di pubblicazione: 1 décembre 2018

Paginazione: 523

ISSN: 0039-2944

\section{Notizia bibliografica digitale}

Francesca Forcolin, «Dominique kalifa, Tu entreras dans le siècle en lisant Fantômas», Studi Francesi

[Online], 186 (LXII | III) | 2018, online dal 01 janvier 2019, consultato il 06 janvier 2021. URL: http:// journals.openedition.org/studifrancesi/15727 ; DOI: https://doi.org/10.4000/studifrancesi.15727

Questo documento è stato generato automaticamente il 6 janvier 2021.

\section{(C) $\odot \Theta$}

Studi Francesi è distribuita con Licenza Creative Commons Attribuzione - Non commerciale - Non opere derivate 4.0 Internazionale. 


\title{
DOMINIQUE KALIFA, Tu entreras dans le siècle en lisant Fantômas
}

\author{
Francesca Forcolin
}

\section{NOTIZIA}

DOMINIQUE KALIFA, Tu entreras dans le siècle en lisant Fantômas, Paris, Vendémiaire, 2017, $329 \mathrm{pp}$.

1 Fantômas, ciclo romanzesco scritto a quattro mani da Pierre Souvestre e Marcel Allain e pubblicato dalle edizioni Fayard tra il 1911 e il 1913, è composto da trentadue volumi che immergono il lettore nell'atmosfera della Belle Époque, dando vita a un incredibile universo letterario. L'opera - eletta dai Surrealisti alla stregua di una creazione letteraria senza precedenti - segna un punto cardine nella storia del romanzo popolare, fondendo due generi, il feuilletton e il poliziesco. Il romanzo tratteggia, con impareggiabile noirceur, le imprese straordinarie di Fantômas, genio del crimine dai mille volti, abile in fughe repentine, apparizioni, trasformazioni e stratagemmi, muovendosi astutamente dai sobborghi londinesi a una Parigi realistica e immaginaria al contempo, sordida, splendida e inquietante, una sorta di città-labirinto. Cendrars, Magritte e Queneau - tra i tanti - hanno celebrato la figura ormai mitica di Fantômas. A dire di Cocteau, l'opera, caratterizzata da un lirismo assurdo e magnifico, può essere considerata come una sorta di epopea, un romanzo collettivo, un'Eneide contemporanea. Dopo la morte di Souvestre, Allain riprese in mano il testo componendo una decina di episodi aggiuntivi che, tuttavia, non si guadagnarono la popolarità dei precedenti. Nei decenni successivi, la figura del ladro gentiluomo e megalomane ha influenzato la cinematografia e la pittura, dando vita a fumetti e opere seriali che ne ripresero i tratti essenziali, quali James Bond o Diabolik.

2 Trentadue, dunque, sono gli episodi canonici, come i capitoli che compongono il volume di Dominique Kalifa: un abecedario di brevi entrées per entrare nel vivo del progetto letterario di Souvestre-Allain, aprendo finestre sul mondo "secondo 
Fantômas" e chiarendo in tal modo numerosi aspetti dell'opera e del mito. Si parte con la A di Apollinaire, uno dei primi intellettuali a scoprire le virtuosità che si nascondono nel personaggio di Fantômas (pp. 11-22); B come Bible (pp. 23-34), a cui il romanzo viene comparato per successo e vendite; segue la C di Capitale (La) (pp.35-43), titolo del giornale dove lavora il reporter Jerome Fandor, che, al pari del poliziotto Juve, ha dichiarato guerra aperta a Fantômas; D di Desnos (pp. 45-51), autore che si è lasciato incantare dall'atmosfera trepidante dell'opera, e alla quale ha dedicato numerosi scritti. Segue E come Epoque (belle?) (pp. 53-61), quella della Parigi dell'immediato preguerra, di cui i volumi sono un perfetto affresco; \& (pp. 63-71), simbolo della proficua unione tra i due autori; Filmographie (pp. 73-85), che conta finora diciassette produzioni; Gurn (pp. 87-92), doppio del bandito, ma senza trucco o travestimenti; H come Haro! (pp.95-101), o coloro che criticarono la scrittura di Souvestre e Allain; Imageries (pp. 103-112), sottolineando la forza visiva dell'opera; Juve (pp.115-123), il poliziotto che si è proposto di porre fine alle malefatte dell'eroe; Kriminal (pp.125-133) come il protagonista, colui che porta una tuta gialla con l'immagine di un teschio, déguisement ripreso da numerosi altri malviventi, tra cui Diabolik; Le Loupart et autres apaches (pp.135-145), capobanda dei Ténébreux, sorta di doppio di Fantômas, ricercato anch'egli dalla giustizia; Méfaits (pp.147-154), gli assassini commessi da Fantômas, enumerati da Sadoul e Prévert durante le serate organizzate intorno al tema, poi elencati in maniera sistematica da Queneau in un capitolo di Bâtons, chiffres et lettres; N come Néanmoins, che rinvia al capitolo Toutefois, il quale ci rimanda a sua volta alla lettera N; Outrances (pp. 157-165), o gli eccessi in cui sconfinano gli autori, passando da una Parigi reale a una fantastica, allucinata, lontana da ogni spiegazione razionale; CEuvres (pp. 167-173), le decine di opere dedicate al leggendario protagonista; $O$, avec ou sans? (pp. 175-179), in riferimento all'accento circonflesso sul nome del protagonista, eliminato da Cendrars e poi di nuovo inserito; Paris (pp. 181-187), la reale e la notturna, che funge da sfondo ai crimini; Phantomas, roi des Belges (pp. 189-196), nome di una rivista surrealista belga degli anni Cinquanta; Queneau (pp. 199-203), uno degli autori che più amarono il romanzo e che contribuì al suo successo; Résurrection (pp. 205-213), tipica caratteristica di un eroe che non muore mai, nemmeno dopo un secolo dalla sua nascita; Société des Amis de Fantômas (pp. 215-229): benché assassino e torturatore, egli vanta un gran numero di ammiratori, lettori anonimi e scrittori, riuniti in associazioni che portano il suo nome; Ubiquité (pp. 233-240), caratteristica degli eroi abili in metamorfosi e travestimenti; Vladimir (pp. 243-249), figlio del protagonista, che con la sua rara presenza partecipa a un risvolto familiare e sentimentale dell'opera; Westwego! (pp. 251-259), espressione che rinvia ai numerosi viaggi, spesso oltrefrontiera; $X$ (pp. 261-269) come erotismo, discreto ma non assente; Yeux (pp. 271-274), l'unica parte visibile dell'eroe quando indossa la maschera, e l'unica del suo corpo a non essere soggetta a trasformazioni; Zigomar et autres Génies du crime (pp. 277-285), opera di Léon Sazie, feuilleton contemporaneo a Fantômas, il cui protagonista è un feroce bandito che incarna i timori della Belle Époque e che, sicuramente, influenzò Souvestre e Allain; ?! (pp. 287-293), i segni di interpunzione che costellano l'opera; * (pp. 295-303), asterisco che rimanda a un argomento che è utile non tralasciare, cioè le molteplici identità che assume Fantômas dopo la sua presunta morte nel naufragio del Gigantic; e ancora, De l'épopée au mythe (pp. 305-310), riflessione sui contorni mitici che hanno fatto di questo genio del crimine un personaggio immortale. Arricchiscono il volume trentadue acquerelli, suddividendo piacevolmente i capitoli. 